\title{
A SINGULAR 1-D HAMILTON-JACOBI EQUATION, WITH APPLICATION TO LARGE DEVIATION OF DIFFUSIONS*
}

\author{
XIAOXUE DENG ${ }^{\dagger}$, JIN FENG ${ }^{\ddagger}$, AND YONG LIU
}

\begin{abstract}
The comparison principle (uniqueness) for the Hamilton-Jacobi equation is usually established through arguments involving a distance function. In this article we illustrate the subtle nature of choosing such a distance function, using a special example of one dimensional Hamiltonian with coefficient singularly (non-Lipschitz) depending upon the state variable. The standard method of using Euclidean distance as a test function fails in such situation. Once the comparison is established, we apply it to obtain a new result on small noise Freidlin-Wentzell type probabilistic large deviation theorem for certain singular diffusion processes.

This article serves to explain basic ideas behind an abstract approach to comparison developed in [J. Feng and T.G. Kurtz, American Mathematical Society, Providence, Rhode Island. Mathematical Surveys and Monographs, 131, 2006], [J. Feng and M. Katsoulakis, Arch. Ration. Mech. Anal., 192(2), 275-310, 2009] in a simple manner, removing all technicalities due to infinite dimensionality.
\end{abstract}

Key words. Comparison principle, singular Hamiltonian, large deviation for singular diffusions.

AMS subject classifications. 49L25, 60F 10.

\section{Introduction}

The comparison principle for the Hamilton-Jacobi equation is usually established through arguments involving a distance function as a test function. When the state space is a subset of Euclidean space and the Hamiltonian has Lipschitz dependence in the state variable, the distance can be chosen to be the usual Euclidean one. This was amply illustrated in the classical theory of viscosity solutions; see, for instance, Crandall, Ishii and Lions [1]. When the Hamiltonian becomes singular, it becomes critical to choose a distance function carefully reflecting intrinsic properties of the Hamiltonian. Lions [7] first pointed out this connection in search of a geometrically invariant condition which gives the comparison principle proof.

Exploring additional special structures of equations, Feng and Katsoulakis [2], Feng and Kurtz [3] introduced an argument utilizing such a distance function idea on examples of the Hamilton-Jacobi equation in the space of measures over $\mathbb{R}^{d}$. In these publications, much technical effort was also devoted to making sense out of a geometrically motivated calculus for functions on the space of measures using modern mass transportation techniques. That may have obscured the overall picture for the Hamilton-Jacobi equation theory. It is therefore of value to expose the key ideas in $[3,2]$ again through new examples in the simplest possible way. In this article, we go to another extreme end by considering the situation of one space dimension.

*Received: April 27, 2010; accepted (in revised version): August 3, 2010. Communicated by Markos Katsoulakis.

The research was partially supported by grants from NSF DMS-0806434, ARO W911NF-08-10064, Kansas TRI KAN0064220, and by Science and Technology Ministry 973 Project of China (2006CB805900), Doctoral Program Foundation of the Ministry of Education of China.

${ }^{\dagger}$ Department of Mathematical Sciences, Tsinghua University, Beijing 100084, People's Republic of China (xdeng.math.tsinghua@gmail.com).

${ }^{\ddagger}$ Department of Mathematics, University of Kansas, Lawrence, KS 66045, USA (jfeng@ math.ku.edu).

$\S$ LMAM, School of Mathematical Sciences, Institute of Mathematics, Peking University, Beijing 100871, People's Republic of China (liuyong@math.pku.edu.cn). 
Let $E:=\mathbb{R}_{+}:=(0, \infty)$; we define a Hamiltonian function $H: \mathbb{R}_{+} \times \mathbb{R} \rightarrow \mathbb{R}$ by

$$
H(x, p):=\kappa(\mu-x) p+\frac{1}{2}|\theta \sqrt{x} p|^{2},
$$

where $\kappa, \mu, \theta>0$ and $2 \kappa \mu>\theta^{2}$. The Hamiltonian is singular in the sense that the $\sqrt{x}$ term is a non-Lipschitz coefficient. In a sense, $x \in E$ can be identified as an unnormalized measure - it is the density of a measure on a fixed point. Analogy between the simplistic 1-D model here and the technically complicated infinite-D models in $[3,2]$ can be drawn based upon this observation.

The main result of this article is a uniqueness type theorem for the nonlinear PDE:

$$
f(x)-\alpha H\left(x, \partial_{x} f(x)\right)=h(x), \quad x \in E, \quad \alpha>0 .
$$

The equation is studied in viscosity sense (to be defined below), which has an implicit dependence on choices of test functions. Such dependence is more pronounced if we write the equation in operator notation

$$
(I-\alpha H) f=h,
$$

where $I$ is the identity map, and $H f(x):=H\left(x, \partial_{x} f(x)\right)$ for $f \in D(H)$, the domain of $H$, with

$$
D(H)=D:=\left\{f: f=g+C, g \in C_{c}^{2}(E), C \in \mathbb{R}\right\} .
$$

Throughout, $C_{c}^{2}(E)$ denotes the collection of functions with compact support in $E$ and with continuous second order derivative, and $C_{b}(E)$ denotes bounded continuous functions on $E$.

THEOREM 1.1. Let $h \in C_{b}(E)$ be uniformly continuous and $D(H)=D$. Then for every bounded upper-semicontinuous viscosity sub-solution $\bar{f}$ and every bounded lower semi-continuous viscosity super-solution $\underline{f}$ of (1.2), both in the sense of Definition 2.2, we have $\bar{f} \leq \underline{f}$.

In particular, the above conclusion implies that there exists at most one bounded continuous viscosity solution to (1.2). Equipped with the above conclusion, we study 1-D diffusion processes with a singular diffusion coefficient:

$$
\left\{\begin{array}{l}
d X_{n}(t)=\kappa\left(\mu-X_{n}(t)\right) d t+n^{-\frac{1}{2}} \theta \sqrt{X_{n}(t)} d W(t), \\
X_{n}(0)>0
\end{array}\right.
$$

This is known as Cox-Ingersoll-Ross model in mathematical finance. The condition $2 \kappa \mu>\theta^{2}$ ensures that there exists a unique $X_{n}(t) \in E$ (the existence and uniqueness for $X_{n}(t) \geq 0$ follows from a result due to Yamada-Watanabe; see Proposition 5.2.13 and Corollary 5.3.23 in Karatzas and Shreve [5]. The strict positivity of $X_{n}(t)$ follows because 0 is a natural boundary; see Chapter 15.6 of Karlin and Taylor [6]).

Next we show that a large deviation principle holds for $\left\{X_{n}(\cdot): n=1,2, \ldots\right\}$. This type of result is along the spirit of Freidlin-Wentzell theory [4] for processes converging to a deterministic trajectory. The novelty here lies in that the diffusion coefficient $\sqrt{x}$ is both non-Lipschitz and degenerate. Therefore, even though the diffusion process $X_{n}$ is simply one dimensional, the theory in [4] and its various generalizations still do not apply. 
Recalling the definition of $H$ in (1.1), we define a Lagrangian $L$ through the Fenchel-Legendre transform

$$
L(x, q):=\sup _{p \in \mathbb{R}}\{p \cdot q-H(x, p)\}=\frac{|q-\kappa(\mu-x)|^{2}}{2 \theta^{2} x} .
$$

By convex duality in Euclidean space, it also follows that

$$
H(x, p)=\sup _{q \in \mathbb{R}}\{p \cdot q-L(x, q)\} .
$$

For a set $A$ in a metric space, we denote $\bar{A}$ and $A^{\circ}$ respectively the closure and interior of the set. We also denote $A C((0, T) ; E)$ the class of $E$-valued absolutely continuous curves on $(0, T)$.

Corollary 1.2. Assume that $\left\{X_{n}(0): n=1,2, \ldots\right\}$ satisfies a large deviation principle with a good rate function $I_{0}$. Then the following holds for all Borel sets $A \subset C([0, T] ; E)$ :

$$
-\inf _{x(\cdot) \in A^{\circ}} I(x(\cdot)) \leq \liminf _{n \rightarrow \infty} \frac{1}{n} \log P\left(X_{n} \in A^{\circ}\right) \leq \limsup _{n \rightarrow \infty} \frac{1}{n} \log P\left(X_{n} \in \bar{A}\right) \leq-\inf _{x(\cdot) \in \bar{A}} I(x(\cdot))
$$

where

$$
I(x)= \begin{cases}I_{0}\left(x_{0}\right)+\int_{0}^{T} L(x(s), \dot{x}(s)) d s, & x(\cdot) \in A C((0, T) ; E) \\ \infty, & \text { otherwise }\end{cases}
$$

Such I has compact level sets and is lower semicontinuous on $C([0, T] ; E)$.

\section{Viscosity solutions and comparison principle}

2.1. Definitions of viscosity solution. First, we define viscosity solution (and strong viscosity solution) for (1.2) with a general operator $H$ in mind (not necessarily that given by (1.1)). We assume that the domain $D(H) \subset M(E)$ and the range $R(H) \subset M(E)$, where $M(E)$ is space of measurable functions on $E$.

DeFINITION 2.1. $\bar{f}$ is a viscosity sub-solution to (1.2), if it is bounded upper semicontinuous and if for every $f_{0} \in D(H)$, there exists $x_{0} \in E$ satisfying both $\left(\bar{f}-f_{0}\right)\left(x_{0}\right)=$ $\sup _{x \in E}\left(\bar{f}-f_{0}\right)(x)$, and $\alpha^{-1}(\bar{f}-h)\left(x_{0}\right) \leq H f_{0}\left(x_{0}\right)$.

$\underline{f}$ is a viscosity super-solution to (1.2), if it is bounded lower semicontinuous, and if for every $f_{1} \in D(H)$, there exists $x_{1} \in E$ satisfying $\left(f_{1}-\underline{f}\right)\left(x_{1}\right)=\sup _{x \in E}\left(f_{1}-\underline{f}\right)(x)$ and $\alpha^{-1}(f-h)\left(x_{1}\right) \geq H f_{1}\left(x_{1}\right)$.

If a continuous function $f$ is both a sub-solution and a super-solution, we call it a viscosity solution.

$\bar{f}$ is called a strong viscosity sub-solution if the following modification is made in the above definition: replace the sentence "there exists $x_{0} \in E$ satisfying ....and ..." by "for every $x_{0} \in E$ satisfying $\left(\bar{f}-f_{0}\right)\left(x_{0}\right)=\sup _{x \in E}\left(\bar{f}-f_{0}\right)(x)$, we have $\alpha^{-1}(\bar{f}-$ $h)\left(x_{0}\right) \leq H f_{0}\left(x_{0}\right)$ ". Similarly, we can define strong viscosity super-solution and strong viscosity solution.

Applied to Equation (1.2) with $D(H)=D$, Definition 2.1 may be vacuous. Since $E$ is not compact, there is no a priori guarantee that there exists those maximizing/minimizing points. To make sense of that definition, one either needs to have growth estimates on $\bar{f}$ and $\underline{f}$ or, equivalently, to alter the class of test functions allowing growth to infinity. 
The following generalized notion of viscosity solution was introduced in [3] for making sense of $(1.2)$ when $D(H)=D$.

Definition 2.2. $\bar{f}$ is a viscosity sub-solution to (1.2), if it is bounded upper semicontinuous and if for every $f_{0} \in D(H)$, there exists a sequence $x_{n} \in E$ satisfying both $\lim _{n \rightarrow \infty}\left(\bar{f}-f_{0}\right)\left(x_{n}\right)=\sup _{x \in E}\left(\bar{f}-f_{0}\right)(x)$, and

$$
\limsup _{n \rightarrow \infty} \alpha^{-1}(\bar{f}-h)\left(x_{n}\right)-H f_{0}\left(x_{n}\right) \leq 0 .
$$

$f$ is a viscosity super-solution to (1.2) if it is bounded lower semicontinuous, and if for every $f_{1} \in D(H)$ there exists a sequence $x_{n} \in E$ satisfying $\lim _{n \rightarrow \infty}\left(f_{1}-\underline{f}\right)\left(x_{n}\right)=$ $\sup _{x \in E}\left(f_{1}-\underline{f}\right)(x)$ and

$$
\liminf _{n \rightarrow \infty} \alpha^{-1}(\underline{f}-h)\left(x_{n}\right)-H f_{1}\left(x_{n}\right) \geq 0 .
$$

2.2. Failure of traditional proof of the comparison principle and the choice of test functions. Formally speaking, the comparison principle follows as a consequence of the maximum principle satisfied by operator $H$. Since the $H$ can only operate on smooth functions and since we have no a priori differentiability estimate on sub- and super- solutions $\bar{f}$ and $f$, an approximate argument is needed; see Chapter 9.1 of [3]. The traditional proof (e.g. Crandall, Ishii and Lions [1]) uses $\sup _{x, y}\left(\bar{f}(x)-\underline{f}(y)-\frac{m}{2}|x-y|^{2}\right)($ as $m \rightarrow \infty)$ to approximate $\sup _{x}(\bar{f}(x)-\underline{f}(x))$. Going through the proof, this requires estimate of the following kind:

$$
H\left(x, \partial_{x} \frac{m}{2}|x-y|^{2}\right)-H\left(y,-\partial_{y} \frac{m}{2}|x-y|^{2}\right) \leq \omega\left(m|x-y|^{2}+|x-y|^{2}\right),
$$

where $\omega(\cdot)$ is a modulus. With the square root singularity in the $\frac{1}{2}|\theta \sqrt{x} p|^{2}$ term of $H(x, p)$, this can never be satisfied.

Lions [7], however, formulates a more general condition with geometric implications of certain first order Hamiltonians in mind. To explain the ideas in a nutshell, we adapt everything to our specific situation. We single out the nonlinear part of $H$ to define $\widetilde{H}(y, p):=\frac{1}{2}|\theta \sqrt{y} p|^{2}$. $\widetilde{H}$ induces a variational distance through its Legendre transform $\widetilde{L}(y, q):=\sup _{p}\{q p-\widetilde{H}(y, p)\}=\frac{1}{2 \theta^{2}} \frac{q^{2}}{y}$. For every $x_{0}, x_{1} \in E$,

$$
\begin{aligned}
d^{2}\left(x_{0}, x_{1}\right) & :=\inf \left\{\int_{0}^{1} \widetilde{L}(y(s), \dot{y}(s)) d s: y(\cdot) \in A C((0,1) ; E), y(0)=x_{0}, y(1)=x_{1}\right\} \\
& =\frac{2}{\theta^{2}}\left|\sqrt{x_{0}}-\sqrt{x_{1}}\right|^{2} .
\end{aligned}
$$

From the explicit calculation $\partial_{x} d^{2}(x, y)=\frac{2}{\theta^{2}}\left(1-\frac{\sqrt{y}}{\sqrt{x}}\right)$ and $\partial_{y} d^{2}(x, y)=\frac{2}{\theta^{2}}\left(1-\frac{\sqrt{x}}{\sqrt{y}}\right)$, we can directly verify the geometric identity

$$
\left|\theta \sqrt{x} \partial_{x} \frac{1}{2} d^{2}(x, y)\right|^{2}=\left|\theta \sqrt{y} \partial_{y} \frac{1}{2} d^{2}(x, y)\right|^{2}=\frac{1}{2} d^{2}(x, y) .
$$

Hence a generalized version of (2.1) holds trivially with $|x-y|$ replaced by $d$ :

$$
\widetilde{H}\left(x, \partial_{x} \frac{m}{2} d^{2}(x, y)\right)-\widetilde{H}\left(y,-\partial_{y} \frac{m}{2} d^{2}(x, y)\right)=0 .
$$


Note that the Cauchy problem $\partial_{t} u=\widetilde{H}\left(x, \partial_{x} u\right)$ has an explicit solution given by the Lax formula

$$
u(t, x):=\sup _{y \in E}\left\{u(0, y)-\frac{d^{2}(x, y)}{t}\right\}
$$

To get a generalized version of (2.1) for $H$ (instead of $\widetilde{H})$, it is sufficient to have

$$
(\mu-x) \partial_{x} d^{2}(x, y)-(\mu-y) \partial_{y}\left(-d^{2}(x, y)\right) \leq 0,
$$

or, in other words, the ODE $\dot{x}=\mu-x$ defines a contraction under $d$. This can be verified directly through computation:

$$
\begin{aligned}
\frac{2}{\theta^{2}}(\mu-x) & \left(1-\frac{\sqrt{y}}{\sqrt{x}}\right)-\left(-\frac{2}{\theta^{2}}(\mu-y)\left(1-\frac{\sqrt{x}}{\sqrt{y}}\right)\right) \\
= & \mu \frac{2}{\theta^{2}}\left(1-\frac{\sqrt{y}}{\sqrt{x}}+1-\frac{\sqrt{x}}{\sqrt{y}}\right)-\frac{2}{\theta^{2}}(x-\sqrt{x y}+y-\sqrt{x y}) \\
= & -\mu \frac{d^{2}(x, y)}{\sqrt{x y}}-\kappa d^{2}(x, y) \leq 0 .
\end{aligned}
$$

There is another problem: to ensure that Definition 2.1 makes sense, we need a certain growth estimate on test functions so that the maximizing/minimizing points can be attained. Specifically, for sub-solutions, we need test functions growing to infinity, and for super-solutions we need test functions growing to negative infinity. This motivates the use of a function $V$ playing a role for the stochastic process $X$ (or a deterministic control problem induced by $H$ ) similar to that played by the Lyapunov function for uncontrolled-ODEs. We would like $V$ to have compact level sets and $\sup _{x} H(x, \partial V(x))<\infty$. Noting that 0 and $+\infty$ are the boundary of $E$ and the dynamics of the stochastic process $X$ behaves differently at these two points, we construct $V$ in three steps. First, let

$$
V_{\infty}(x)=\varepsilon \log (1+U(x)), \quad U(x)=\frac{|\mu-x|^{2}}{2}, \quad \varepsilon>0 .
$$

This function has the property that, when $\varepsilon$ is small enough,

$$
\sup _{x} H\left(x, \partial_{x} V_{\infty}(x)\right)=\sup _{x}\left\{-\varepsilon\left(1-\frac{\varepsilon}{2} \frac{\theta^{2} x}{1+U(x)}\right) \frac{|\partial U(x)|^{2}}{1+U(x)}\right\} \leq 0 .
$$

Now consider $V_{0}(x)=-\log x$; then

$$
\sup _{x} H\left(x, \partial_{x} V_{0}(x)\right)=\sup _{x}\left\{-\frac{1}{x}\left(\kappa \mu-\frac{1}{2} \theta^{2}\right)+\kappa\right\} \leq \kappa<\infty,
$$

where we used critically the condition $2 \kappa \mu>\theta^{2}$. Finally, we smoothly paste $V_{0}$ and $V_{\infty}$ by

$$
V(x):=\varphi(x) V_{0}(x)+(1-\varphi(x)) V_{\infty}(x),
$$

where $\varphi \in C^{\infty}$ satisfies $\varphi^{\prime} \leq 0$ and

$$
\varphi(y)= \begin{cases}1, & y \in(0,1), \\ 0, & y \in(2, \infty) .\end{cases}
$$


It follows then $V(x)$ has compact level sets in $E$ and $\sup _{x} H\left(x, \partial_{x} V(x)\right)<\infty$.

Using the above inequality, by convexity of $H(x, p)$ in $p$, we now introduce two new classes of test functions: for $0<\epsilon \leq 1$ and $m>0$, define

$$
\begin{aligned}
& D_{0}:=\left\{f_{0}: f_{0}(\cdot):=(1-\epsilon) m d^{2}(\cdot, y)+\epsilon V(\cdot)+\varphi(\cdot), \quad \varphi \in D, y \in E\right\}, \\
& D_{1}:=\left\{f_{1}: f_{1}(\cdot):=-(1+\epsilon) m d^{2}(x, \cdot)-\epsilon V(\cdot)+\varphi(\cdot), \quad \varphi \in D, x \in E\right\},
\end{aligned}
$$

and two more operators

$$
\begin{array}{ll}
H_{0} f_{0}(x):=H\left(x, \partial_{x} f_{0}(x)\right), & f_{0} \in D_{0}, \\
H_{1} f_{1}(x):=H\left(x, \partial_{x} f_{1}(x)\right), & f_{1} \in D_{1} .
\end{array}
$$

More discussion on such test functions can be found in detail in Section 1.2.2 on page 285 of $[2]$.

It follows that $H_{0} f_{0}, H_{1} f_{1} \in C(E)$ are well defined for all $x \in E$. We will prove the comparison between the sub-solution given by $H_{0}$ and the super-solution given by $H_{1}$ in Lemmas 2.4 and 2.5. Then, by linking respective sub- and super-solutions of $H_{0}$ and $H_{1}$ with those of $H$ in Lemma 2.6, we arrive at the conclusion of Theorem 1.1. $H_{0}$ and $H_{1}$ can be viewed as sub- and super- extensions from the $H$ in (1.2), which is defined only on $D$ (Lemma 2.6).

2.3. Comparison principle for some related equations. The domain $D$ of $H$ only contains smooth functions with compact supports. This class is easier to work with when one is performing convergence type analysis, using for instance the Barles-Perthame half-relaxed limit approach. However, for the proof of uniqueness (comparison principle), we need test functions in $D_{0}$ and $D_{1}$. First, we prove an important property which generalizes (2.1):

LEMMA 2.3.

$$
\begin{aligned}
\frac{1}{1-\epsilon}( & \left.H_{0}\left((1-\epsilon) m d^{2}(\cdot, y)+\epsilon V(\cdot)\right)(x)\right)-\frac{1}{1+\epsilon}\left(H_{1}\left(-(1+\epsilon) m d^{2}(x, \cdot)-\epsilon V(\cdot)\right)(y)\right) \\
\leq & \left(\frac{\epsilon}{1-\epsilon}+\frac{\epsilon}{1+\epsilon}\right) \sup _{x} H\left(x, \partial_{x} V(x)\right) .
\end{aligned}
$$

Proof. By convexity of $H(x, p)$ in $p$,

$$
\begin{aligned}
H_{0}\left((1-\epsilon) m d^{2}(\cdot, y)+\epsilon V(\cdot)\right)(x) \\
\quad \leq(1-\epsilon) H\left(x, \partial_{x} m d^{2}(x, y)\right)+\epsilon H\left(x, \partial_{x} V(x)\right) \\
\quad \leq(1-\epsilon)\left[\kappa m \frac{2}{\theta^{2}}(\mu-x)\left(1-\frac{\sqrt{y}}{\sqrt{x}}\right)+m^{2} \frac{2}{\theta^{2}}(\sqrt{y}-\sqrt{x})^{2}\right]+\epsilon H\left(x, \partial_{x} V(x)\right) .
\end{aligned}
$$

Similarly,

$$
\begin{aligned}
& H_{1}\left(-(1+\epsilon) m d^{2}(x, \cdot)-\epsilon V(\cdot)\right)(y) \\
& \quad \geq(1+\epsilon)\left[-\kappa m \frac{2}{\theta^{2}}(\mu-y)\left(1-\frac{\sqrt{x}}{\sqrt{y}}\right)+m^{2} \frac{2}{\theta^{2}}(\sqrt{y}-\sqrt{x})^{2}\right]-\epsilon H\left(y, \partial_{y} V(y)\right) .
\end{aligned}
$$

By (2.3), the conclusion follows. 
With the above estimate, the next lemma follows by modifying the standard arguments in [1].

LEMma 2.4. Let $h_{0}, h_{1} \in C_{b}(E)$. Let bounded upper semicontinuous $\bar{f}$ be a strong sub-solution of

$$
\left(I-\alpha H_{0}\right) \bar{f}=h_{0},
$$

and bounded lower semicontinuous $\underline{f}$ be a strong super-solution of

$$
\left(I-\alpha H_{1}\right) \underline{f}=h_{1},
$$

both in the sense of Definition 2.1. Then

$$
\sup _{x \in E}\{\bar{f}(x)-\underline{f}(x)\} \leq \sup _{x \in E}\left\{h_{0}(x)-h_{1}(x)\right\} .
$$

Proof. Let

$$
\Phi_{\epsilon, m}(x, y)=\frac{1}{1-\epsilon} \bar{f}(x)-\frac{1}{1+\epsilon} f(y)-m d^{2}(x, y)-\frac{\epsilon}{1-\epsilon} V(x)-\frac{\epsilon}{1+\epsilon} V(y),
$$

where $0<\epsilon<1$ and $m>1$. Since $V$ has compact level sets, there exists $\left(x_{\epsilon, m}, y_{\epsilon, m}\right) \epsilon$ $E \times E$ satisfying

$$
\Phi_{\epsilon, m}\left(x_{\epsilon, m}, y_{\epsilon, m}\right)=\sup _{(x, y) \in E \times E} \Phi_{\epsilon, m}(x, y) .
$$

Let $f_{0}(x)=(1-\epsilon) m d^{2}\left(x, y_{\epsilon, m}\right)+\epsilon V(x)$; then

$$
\bar{f}\left(x_{\epsilon, m}\right)-f_{0}\left(x_{\epsilon, m}\right)=\sup _{x \in E}\left\{\bar{f}(x)-f_{0}(x)\right\} .
$$

By the strong sub-solution property of $\bar{f}, \quad \alpha^{-1}\left(\bar{f}-h_{0}\right)\left(x_{\epsilon, m}\right) \leq\left(H_{0} f_{0}\right)\left(x_{\epsilon, m}\right)$. Similarly, let $f_{1}(y)=(1+\epsilon) m d^{2}\left(x_{\epsilon, m}, y\right)-\epsilon V(y)$; then $\alpha^{-1}\left(\underline{f}-h_{1}\right)\left(y_{\epsilon, m}\right) \geq\left(H_{1} f_{1}\right)\left(y_{\epsilon, m}\right)$. Thus,

$$
\begin{aligned}
\sup _{x \in E}\{ & \left.\frac{1}{1-\epsilon} \bar{f}(x)-\frac{1}{1+\epsilon} \underline{f}(x)-\frac{2 \epsilon}{1-\epsilon^{2}} V(x)\right\} \\
= & \sup _{x \in E} \Phi_{\epsilon, m}(x, x) \\
\leq & \Phi_{\epsilon, m}\left(x_{\epsilon, m}, y_{\epsilon, m}\right) \\
\leq & \frac{1}{1-\epsilon} \bar{f}\left(x_{\epsilon, m}\right)-\frac{1}{1+\epsilon} f\left(y_{\epsilon, m}\right)-\left(\frac{\epsilon}{1-\epsilon}+\frac{\epsilon}{1+\epsilon}\right) \inf _{x} V(x) \\
\leq & \alpha\left[\frac{1}{1-\epsilon}\left(H_{0} f_{0}\left(x_{\epsilon, m}\right)\right)-\frac{1}{1+\epsilon}\left(H_{1} f_{1}\left(y_{\epsilon, m}\right)\right)\right] \\
& +\left(h_{0}\left(x_{\epsilon, m}\right)-h_{1}\left(y_{\epsilon, m}\right)\right)+\alpha\left(\frac{\epsilon}{1-\epsilon}\left\|h_{0}\right\|_{\infty}+\frac{\epsilon}{1+\epsilon}\left\|h_{1}\right\|_{\infty}\right) \\
& \quad-\left(\frac{\epsilon}{1-\epsilon}+\frac{\epsilon}{1+\epsilon}\right) \inf _{x} V(x) .
\end{aligned}
$$

By a standard argument (e.g. Lemma 9.2 in [3] or Proposition 3.7 of [1]),

$$
\lim _{m \rightarrow \infty} m d^{2}\left(x_{\epsilon, m}, y_{\epsilon, m}\right)=0 .
$$

Together with Lemma 2.3, taking $\lim _{\epsilon \rightarrow 0+} \lim _{m \rightarrow \infty}$, we arrive at the conclusion. 
2.4. Comparison principle for Equation (1.2). $\quad H_{0}, H_{1}, H$ are local operators. Similar to the proof of Lemma 9.9 of [3], we have the following.

LEMma 2.5. Any sub-solution of (2.6) is also a strong sub-solution of (2.6), and any super-solution of (2.7) is also a strong super-solution of (2.7); all in the sense of Definition 2.1.

In view of 2.4 and 2.5 , we are only one step away from concluding Theorem 1.1. This step is provided in the following lemma.

Lemma 2.6. Any sub-solution to (1.2), in the sense of Definition 2.2, is also a subsolution to $f-\alpha H_{0} f=h$ in the sense of Definition 2.1. Similarly, any super-solution to (1.2) is also a super-solution to $f-\alpha H_{1} f=h$.

Proof. We only prove the sub-solution case. The case of super-solution is similar.

Let $\bar{f}$ be a sub-solution to (1.2) and $f_{0} \in D_{0}$. By the definition of $D_{0}$, and the facts that $\lim _{x \rightarrow 0+} f_{0}(x)=\infty$ and $\lim _{x \rightarrow+\infty} f_{0}(x)=\infty$, there exists $0<a<b<\infty$ such that $f_{0}(a)=f_{0}(b)=C$ and

$$
\sup _{x \in[a, b]}\left\{\bar{f}(x)-f_{0}(x)\right\}=\sup _{x \in E}\left\{\bar{f}(x)-f_{0}(x)\right\} .
$$

Moreover, for $\forall x \notin[a, b], f(x)>C$ and

$$
\sup _{x \in E} \bar{f}(x)-C<\sup _{x \in E}\left\{\bar{f}(x)-f_{0}(x)\right\} .
$$

After taking $\left\{a_{n}\right\}_{n=1}^{\infty}$ such that $0<a_{n}<a, a_{n} \downarrow 0$, and $\left\{b_{n}\right\}_{n=1}^{\infty}$ such that $b_{n}>b, b_{n} \uparrow \infty$, we construct $f_{n} \in D$ satisfying

$$
\begin{cases}f_{n}(x)=f_{0}(x), & x \in\left[a_{n}, b_{n}\right], \\ f_{0}\left(a_{n}\right)<f_{n}(x)<f_{0}\left(a_{n}\right)+1, & x \in\left(a_{n+1}, a_{n}\right), \\ f_{0}\left(b_{n}\right)<f_{n}(x)<f_{0}\left(b_{n}\right)+1, & x \in\left(b_{n}, b_{n+1}\right), \\ f_{n}(x)=f_{0}\left(a_{n}\right)+1, & x \in\left(0, a_{n+1}\right], \\ f_{n}(x)=f_{0}\left(b_{n}\right)+1, & x \in\left[b_{n+1}, \infty\right) .\end{cases}
$$

Hence, by the definition of sub-solution to (1.2), there exists a sequence $\left\{x_{n}\right\}_{n=1}^{\infty} \subset$ $[a, b]$ satisfying

$$
\bar{f}\left(x_{n}\right)-f_{n}\left(x_{n}\right) \geq \sup _{x \in E}\left\{\bar{f}(x)-f_{n}(x)\right\}-\frac{1}{2^{n}}
$$

which implies

$$
\limsup _{n \rightarrow \infty}\left(\alpha^{-1}(\bar{f}-h)\left(x_{n}\right)-H f_{n}\left(x_{n}\right)\right) \leq 0 .
$$

Therefore there is a convergent subsequence $x_{n_{k}} \rightarrow x_{0}$ as $k \rightarrow \infty$. Then

$$
\limsup _{k \rightarrow \infty}\left((\bar{f}-h)\left(x_{n_{k}}\right)\right) \leq \limsup _{k \rightarrow \infty} \alpha H f_{n_{k}}\left(x_{n_{k}}\right)=\alpha H_{0} f_{0}\left(x_{0}\right) .
$$

By (2.9), we have

$$
\limsup _{k \rightarrow \infty} \bar{f}\left(x_{n_{k}}\right)-\liminf _{k \rightarrow \infty} f_{n_{k}}\left(x_{n_{k}}\right) \geq \liminf _{k \rightarrow \infty}\left(\bar{f}\left(x_{n_{k}}\right)-f_{n_{k}}\left(x_{n_{k}}\right)\right) \geq \bar{f}\left(x_{0}\right)-f_{0}\left(x_{0}\right),
$$

which implies that $\limsup _{k \rightarrow \infty} \bar{f}\left(x_{n_{k}}\right) \geq f_{0}\left(x_{0}\right)$. By upper semicontinuity of $\bar{f}$, $\limsup _{k \rightarrow \infty} \bar{f}\left(x_{n_{k}}\right)=f\left(x_{0}\right)$. Therefore, the conclusion follows. 


\section{Large deviation for singular diffusions}

We consider a sequence of processes $\left\{X_{n}(\cdot): n=1,2, \ldots\right\}$ given by stochastic differential Equation (1.3). By Ito's formula, the generator of $X_{n}$ is given by

$$
A_{n} f(x)=\kappa(\mu-x) \partial_{x} f(x)+\frac{1}{2 n} \theta^{2} x \partial_{x x} f(x), \quad f+c \in C_{c}^{2}(E), \quad c \in \mathbb{R} .
$$

Following [3] (e.g. Theorem 2.10 or 7.18, and Corollary 8.28), large deviation properties of $\left\{X_{n}(\cdot): n=1,2, \ldots\right\}$ can be established by studying

$$
H_{n} f(x):=\frac{1}{n} e^{-n f} A_{n} e^{n f}(x)=\kappa(\mu-x) \partial_{x} f(x)+\frac{1}{2}\left|\theta \sqrt{x} \partial_{x} f\right|^{2}+\frac{1}{2 n} \theta^{2} x \partial_{x x} f, \quad f \in D .
$$

The following convergence result follows by direct verification.

Lemma 3.1. For every $f \in D$,

$$
\lim _{n \rightarrow+\infty}\left\|H_{n} f-H f\right\|_{\infty}=0 .
$$

In order to apply Theorem 2.10 in [3], we also need to verify an exponential compact containment type condition (Condition 2.8 of [3]) for the processes. This is verified in the following.

With a slight abuse of notation, we make obvious extension of $H_{n} f(x)$ even when $f \in C^{2}(E)$ but $f \notin D$. First, under the condition $2 \kappa \mu>\theta^{2}$, when $N$ is large enough

$$
\sup _{n \geq N x \in E} \sup _{n} V_{0}(x)=\sup _{n \geq N x \in E}\left\{-\frac{1}{2 x}\left(2 \kappa \mu-\theta^{2}\left(1+\frac{1}{n}\right)\right)\right\}+\kappa \leq \kappa<\infty .
$$

Similar to the verification of $\sup _{x \in E} H\left(x, \partial_{x} V(x)\right)<\infty$, we have

$$
\gamma:=\sup _{n \geq N} \sup _{x \in E} H_{n} V(x)<\infty .
$$

Lemma 3.2. For each compact set $K_{0} \subset \subset E$, and each $\alpha>0$, there exists another compact set $K_{\alpha} \subset \subset E$ such that

$$
\limsup _{n \rightarrow \infty} \frac{1}{n} \log \sup _{x \in K_{0}} P\left(\exists t \leq T, X_{n}(t) \notin K_{\alpha} \mid X_{n}(0)=x\right) \leq-\alpha .
$$

Proof. Apply Ito's formula, it follows that

$$
M_{n}(t):=e^{n\left[V\left(X_{n}(t)\right)-V\left(X_{n}(0)\right)-\int_{0}^{t} H_{n} V\left(X_{n}(s)\right) d s\right]}
$$

is a super-martingale. Choose a compact set $K_{1} \subset \subset E, K_{1} \supset K_{0}$, and define

$$
\tau_{n}:=\inf \left\{t>0, X_{n}(t) \notin K_{1}\right\}
$$

and

$$
\beta:=\inf _{y \in K_{1}^{c}} V(y)-\sup _{y \in K_{0}} V(y) .
$$

By the optional stopping theorem, for $n \geq N$,

$$
P\left(\exists t \leq T, X_{n}(t) \notin K_{1} \mid X_{n}(0)=x\right) e^{n[\beta-T \gamma]} \leq E\left[M_{n}\left(T \wedge \tau_{n}\right) \mid X_{n}(0)=x\right]=1 .
$$


That is,

$$
\sup _{x \in K_{0}} P\left(\exists t \leq T, X_{n}(t) \notin K_{1} \mid X_{n}(0)=x\right) \leq e^{-n[\beta-T \gamma]} .
$$

For the given $\alpha>0$, since $\lim _{M \rightarrow \infty} \inf _{|x| \geq M} V(x)=+\infty$, we have the conclusion by choosing $\beta$ sufficiently large.

With Lemmas 3.1 and 3.2, combined with the comparison principle for $H$ in Theorem 1.1, by Theorem 2.10 (or 7.18 of [3]) we have that $\left\{X_{n}: n=1,2, \ldots\right\}$ satisfies the large deviation principle. Next, we explicitly identify its rate function $I$ and show that it is given as in (1.6).

Recalling the definitions of $H(x, p)$ and $L(x, q)$ in (1.5) and (1.4), we have the following variational representation of $H$ :

$$
H f(x)=\sup _{u \in \mathbf{R}}\left\{u \cdot \partial_{x} f(x)-L(x, u)\right\}, \quad f \in D .
$$

For readers familiar with optimal control theory, the above implies that the operator $H$ is a generator of a Nisio semigroup corresponding to the simple controlled ODE $\dot{x}(t)=u(t)$ with running cost $I_{T}[x(\cdot)]=\int_{0}^{T} L(x(s), u(s)) d s$.

In order to apply Corollary 8.28 of [3], first we verify Condition 8.9 in that book. Conditions 8.9.1-8.9.3 hold trivially. Condition 8.9.4 requires that, for each compact set $K \subset \subset E$ and $0 \leq M<\infty$, there exists a compact set $\hat{K} \subset \subset E$ such that for any trajectory $x(\cdot), x(0) \in K$ and $\int_{0}^{T} L(x, \dot{x}) d s \leq M$ imply that $x(t) \in \hat{K}, 0 \leq t \leq T$. To see that this holds, we note that such $x(\cdot)$ is absolutely continuous and that $x(s)>0$ for each $s>0$. Let $u(t)=\frac{\dot{x}(t)-\kappa(\mu-x(t))}{\theta \sqrt{x(t)}}$ - that is, $\dot{x}(t)=\kappa(\mu-x(t))+\theta \sqrt{x(t)} u(t)$; then

$$
\begin{aligned}
\frac{d}{d t} V_{0}(x(t)) & =-\frac{\dot{x}(t)}{x(t)} \\
& =-\frac{1}{x(t)}(\kappa \mu-\theta \sqrt{x(t)} u(t))+\kappa \\
& =-\frac{1}{x(t)}\left(\kappa \mu-\frac{\theta^{2}}{2}\right)+\left(\frac{\theta^{2}}{2}-\theta \sqrt{x(t)} u(t)\right)\left(-\frac{1}{x(t)}\right)+\kappa \\
& \leq-\frac{\theta^{2}}{2} \frac{1}{x(t)}+\frac{\theta}{x(t)} u(t)+\kappa \\
& \leq \frac{u^{2}(t)}{2}+\kappa
\end{aligned}
$$

Therefore,

$$
V_{0}(x(t))-V_{0}(x(0)) \leq \frac{1}{2} \int_{0}^{T}|u(t)|^{2} d t+\kappa T .
$$

On the other hand, there exists a constant $C$ such that

$$
\begin{aligned}
\frac{d}{d t} V_{\infty}(x(t)) & =\epsilon \frac{\partial_{x} U(x(t))}{1+U(x(t))} \dot{x}(t) \\
& =\epsilon \frac{-[\kappa(\mu-x(t))]^{2}}{1+\frac{\kappa(\mu-x(t))^{2}}{2}}+\epsilon \frac{-\kappa(\mu-x(t))}{1+\frac{\kappa(\mu-x(t))^{2}}{2}} \theta \sqrt{x(t)} u(t) \\
& \leq \epsilon \frac{-\kappa(\mu-x(t))}{1+\frac{\kappa(\mu-x(t))^{2}}{2}} \theta \sqrt{x(t)} u(t) \leq C|u(t)|
\end{aligned}
$$


That is,

$$
V_{\infty}(x(t))-V_{\infty}(x(0)) \leq C T \int_{0}^{T}|u(t)|^{2} d t .
$$

Together, these estimates imply that

$$
V(x(t))-V(x(0)) \leq C T \int_{0}^{T}|u(t)|^{2} d t+\kappa T .
$$

By properties of $V$, Condition 8.9.4 in [3] is satisfied as well.

For each $f \in C_{c}^{2}(E)$ and compact set $K \subset \subset E$, there is a constant $C_{K, f}$ such that

$$
\left|u \cdot \partial_{x} f(x)\right| \leq C_{K, f}(L(x, u))^{\frac{1}{2}+\epsilon}, \quad \forall(x, u) \in K \times U .
$$

Thus Condition 8.9.5 holds.

Next, we verify Condition 8.10 in [3] by selecting a trajectory $x(\cdot)$ satisfying $x(0)=x_{0}>0$, and for every $t>0, x(t)>0$ and

$$
\int_{0}^{T} L(x, \dot{x}) d s=\int_{0}^{T} \frac{|\dot{x}(s)-\kappa(\mu-x(s))|^{2}}{2 \theta^{2} x(s)} d s=0 .
$$

The solution to the ODE

$$
\dot{x}(t)=\kappa(\mu-x(t)), \quad x(0)=x_{0}
$$

satisfies the requirement.

Finally, Condition 8.11 in [3] requires us, for each $f \in D$, to find an $x(\cdot) \in$ $C([0, T] ; E)$ such that

$$
\int_{t_{1}}^{t_{2}} H f(x(s)) d s=\int_{t_{1}}^{t_{2}}\left(\dot{x}(s) \cdot \partial_{x} f(x(s))-L(x(s), \dot{x}(s))\right) d s .
$$

Since

$$
H(x, p)=\partial_{p} H(x, p) p-L\left(x, \partial_{p} H(x, p)\right),
$$

where $\partial_{p} H(x, p)=\kappa(\mu-x)+\theta^{2} x p$, the above condition is satisfied for any solution to the ODE

$$
\dot{x}(t)=\kappa(\mu-x(t))+\theta^{2} x(t) \partial_{x} f(x(t)) .
$$

Existence of a solution $x_{f}(t)>0, t \in[0, T]$ to the above equation follows from standard theory. Therefore Condition 8.11 in [3] holds as well.

By Theorem 8.14 and Corollary 8.28 of $[3]$, the rate function of $\left\{X_{n}(\cdot): n=1,2, \ldots\right\}$ admits the form given by Corollary 1.2.

\section{REFERENCES}

[1] M.G. Crandall, H. Ishii and P.-L. Lions, User's guide to viscosity solutions of second order partial differential equations, Bulletin of the A.M.S., 27(1), 1-67, 1992.

[2] J. Feng and M. Katsoulakis, A comparison principle for Hamilton-Jacobi equations related to controlled gradient flows in infinite dimensions, Arch. Ration. Mech. Anal., 192(2), 275-310, 2009. 
[3] J. Feng and T.G. Kurtz, Large Deviations for Stochastic Processes, American Mathematical Society, Providence, Rhode Island. Mathematical Surveys and Monographs, 131, 2006.

[4] M.I. Freidlin and A.D. Wentzell, Random Perturbations of Dynamical Systems, Translated from the 1979 Russian original by Joseph Szcs,Second edition, Fundamental Principles of Mathematical Sciences, Springer-Verlag, New York, 260, 1998.

[5] I. Karatzas and S.E. Shreve, Brownian Motion and Stochastic Calculus, Second Edition, Springer, New York, 1991.

[6] S. Karlin and H.M. Taylor, A Second Course in Stochastic Processes, Academic Press, New York, 1981.

[7] P.-L. Lions, Some properties of the viscosity semigroups for Hamilton-Jacobi equations, Nonlinear differential equations (Granada, 1984), Res. Notes in Math., J.K. Hale and P. MartinezAmores, Eds., Pitman, Boston, MA, 132, 43-63, 1985. 